\title{
Preparation of PU/Fibrin Vascular Scaffold with Good Biomechanical Properties and Evaluation of Its Performance in vitro and in vivo
}

This article was published in the following Dove Press journal: International Journal of Nanomedicine

\author{
Lei Yang ${ }^{1,2, *}$ \\ Xiafei $\mathrm{Li}^{3, *}$ \\ Yiting $\mathrm{Wu}^{4}$ \\ Pengchong $\mathrm{Du}^{1,5}$ \\ Lulu Sun' \\ Zhenyang $\mathrm{Yu}^{2}$ \\ Shuang Song' \\ Jianshen Yin' \\ Xianfen $\mathrm{Ma}^{\mathrm{I}}$ \\ Changqin Jing' \\ Junqiang Zhao ${ }^{3}$ \\ Hongli Chen' \\ Yuzhen Dong ${ }^{2}$ \\ Qiqing Zhang' \\ Liang Zhao ${ }^{1,6,7}$
}

'College of Life Science and Technology, Xinxiang Medical University, Xinxiang, People's Republic of China; ${ }^{2}$ Department of Orthopedics, First Affliated Hospital, Xinxiang Medical University, Xinxiang, People's Republic of China; ${ }^{3}$ College of Medical Engineering, Xinxiang Medical University, Xinxiang, People's Republic of China; ${ }^{4}$ Xiacun Community Health Service Center, Shenzhen Hospital, University of Chinese Academy of Sciences, Shenzhen, People's Republic of China; ${ }^{5}$ Department of Cardio-Thoracic Surgery, Third Affiliated Hospital, Xinxiang Medical University, Xinxiang, People's Republic of China; ${ }^{6} \mathrm{Key}$ Laboratory of Cardiac Structure Research, Zhengzhou Seventh People's Hospital, Zhengzhou, People's Republic of China; ${ }^{7} T$ The Central Lab, The Third People's Hospital of

Datong, Datong, People's Republic of China

*These authors contributed equally to this work

Correspondence: Liang Zhao;

Yuzhen Dong

Email zhaoliang4321@163.com;

dongyuzhen1998@163.com
Purpose: The development of tissue-engineered blood vessels provides a new source of donors for coronary artery bypass grafting and peripheral blood vessel transplantation. Fibrin fiber has good biocompatibility and is an ideal tissue engineering vascular scaffold, but its mechanical property needs improvement.

Methods: We mixed polyurethane (PU) and fibrin to prepare the PU/fibrin vascular scaffolds by using electrospinning technology in order to enhance the mechanical properties of fibrin scaffold. We investigated the morphological, mechanical strength, hydrophilicity, degradation, blood and cell compatibility of PU/fibrin (0:100), PU/fibrin (5:95), PU/fibrin $(15: 85)$ and PU/fibrin (25:75) vascular scaffolds. Based on the results in vitro, PU/fibrin $(15: 85)$ was selected for transplantation in vivo to repair vascular defects, and the extracellular matrix formation, vascular remodeling, and immune response were evaluated.

Results: The results indicated that the fiber diameter of the PU/fibrin (15:85) scaffold was about $712 \mathrm{~nm}$. With the increase of PU content, the mechanical strength of the composite scaffolds increased, however, the degradation rate decreased gradually. The PU/fibrin scaffold showed good hydrophilicity and hemocompatibility. PU/fibrin (15:85) vascular scaffold could promote the adhesion and proliferation of mesenchymal stromal cells (MSCs). Quantitative RT-PCR experimental results showed that the expression of collagen, survivin and vimentin genes in PU/fibrin (15:85) was higher than that in PU/fibrin (25:75). The results in vivo indicated the mechanical properties and compliance of PU/fibrin grafts could meet clinical requirements and the proportion of thrombosis or occlusion was significantly lower. The graft showed strong vasomotor response, and the smooth muscle cells, endothelial cells, and ECM deposition of the neoartery were comparable to that of native artery after 3 months. At 3 months, the amount of macrophages in PU/fibrin grafts was significantly lower, and the secretion of pro-inflammatory and anti-inflammatory cytokines decreased.

Conclusion: PU/fibrin (15:85) vascular scaffolds had great potential to be used as smalldiameter tissue engineering blood vessels.

Keywords: PU/fibrin scaffold, small diameter, in vitro biocompatibility, biomechanical properties, vascular remodeling

\section{Introduction}

Cardiovascular diseases (CVDs) have always threatened the physical and mental health of human beings worldwide. According to the results of the World Health Organization research report, the morbidity and mortality of CVDs are significantly higher than other types of diseases. ${ }^{1}$ At the same time, the number of deaths from CVDs accounts for about $30.8 \%$ of all diseases deaths in the United States, and the 
researchers predict that the number of CVDs will reach 23.4 million in the next 15 years. ${ }^{2,3}$ To date, the majority of medical researchers generally believe that the best treatment for serious CVDs is vascular reconstruction. In detail, the main surgical methods based on the severity of CVDs are percutaneous coronary intervention or coronary artery bypass grafting. Simultaneously, the incidence rate of pediatric congenital cardiovascular defects (CCDs) in the United States is $0.83 \%$, such as atrial septal defect, ventricular septal defect and patent ductus arteriosus. Most pediatric patients with CVDs due to CCDs should be repeatedly treated with reconstructive surgery. ${ }^{4}$ Moreover, blood vessels in the bypass treatment of CVDs and CCDs usually come from patient's own saphenous veins. Due to small size, varicosities, anatomic variation, previous harvesting, or other abnormalities in autologous transplantation, so its application is limited. ${ }^{5}$ Therefore, the emergence of artificial vascular grafts brings a new feasible and effective method for the treatment of various types of arterial complications, especially suitable for young patients.

In recent years, with the joint efforts of researchers around the world, tissue engineering vascular grafts (TEVGs) have obtained rapid progress. Large-diameter artificial blood vessels $(>6 \mathrm{~mm}$ ) have demonstrated significant therapeutic effects in the clinic. However, it is disappointing that small-diameter artificial vascular grafts $(<6 \mathrm{~mm})$, such as the inguinal arteries and coronary arteries transplantation, have not yet achieved satisfactory results until now. ${ }^{6}$ This is due to the low blood velocity in the small-diameter artificial vascular graft causes thrombosis and intimal hyperplasia, which in turn causes stenosis of vessel lumen, resulting in insufficient patency. ${ }^{7}$ Thus, it is urgent to prepare a small-diameter artificial vascular graft similar to the inherent structure of native artery.

Fibrin belongs to a kind of natural biological scaffold material. It is derived from plasma proteins in human blood. It shows no immune rejection reaction, good biocompatibility, degradability and high affinity to biological surfaces. ${ }^{8}$ Furthermore, fibrin can also combine well with cells, which is beneficial to cell growth. ${ }^{9}$ Unfortunately, fibrin exhibits poor mechanical strength and often cannot be used alone as a vascular scaffold material. ${ }^{10}$ Polyurethane (PU) is a polymer synthetic material. It was first prepared by German scientist Otto Bayer in 1937 using hexamethylene diisocyanate and hexamethylenediamine. It exhibits good mechanical strength, good biocompatibility and easy processing, so it has been widely used in biomedical materials and can be used as a suitable small-diameter tissue engineering vascular scaffold material. ${ }^{11,12}$

Currently, the ideal mechanical properties and hydrophilicity in the preparation of artificial vascular scaffolds are essential factors. The scaffold material with good mechanical strength can be sufficient to resist the impact of blood flow and ensure that it does not bend or break. At the same time, a hydrophilic scaffold can promote cell proliferation and adhesion, and also improve the role of vascular reconstruction and remodeling in vivo. ${ }^{13}$ In order to make up for the shortcomings of various materials and make full use of their advantages. The researchers successfully blended native materials and polymer materials to build an excellent composite material.

In this work, we fabricated a PU/fibrin tubular graft by electrospinning technology, with PU with strong mechanical properties and fibrin with good affinity. Then, we evaluated its physicochemical, mechanical properties, hemocompatibility, degradation in vitro and cell compatibility. Then, we explored the function, mechanical, histological and immunological analysis of PU/fibrin (15:85) vascular grafts in vivo.

\section{Materials and Methods Materials}

PU was purchased from Sigma-Aldrich (Cat No.81367-5G, America). Fibrin was provided by Thermo Scientific company (Cat No.MA126074, America). Ninety-eight percent formic acid was provided by Aladdin company (Cat No. F112034, China). Dulbecco's modified eagle's medium (DMEM), phosphate buffer saline (PBS, Cat No.P1022500), fetal bovine serum (FBS, Cat No.S9030-200), $\mathrm{CaCl}_{2}$ (Cat No.G0071) and lipase (Cat No.L8621) were from Solarbio (Beijing, China). Living cell staining kit (Cat No. C2015S), CCK-8 kit (Cat No.C0038), ELISA kit (Cat No. PI328), KCL (Cat No.ST1596) were purchased from Beyotime. Sodium nitroprusside (SNP, Cat No.S817931), adrenaline (AD, Cat No.N814761) and acetylcholine (Ach, Cat No.A886059) were obtained from Macklin (Shanghai, China). Anti-von Willebrand Factor antibody (ab115771), Anti- $\alpha$-SMA (251411), Mouse Anti-CD68 antibody were obtained from Ruizekang Biotechnology Co., Ltd. (Beijing, China). Safranin O (Cat No.G1031), Verhoeff (Cat No. G1046) and Sirius red (Cat No.G1038) kit were supplied by Servicebio Biochemical Technology Co., Ltd 
(Wuhan, China). Mesenchymal stromal cells (MSCs) were obtained from Xinxiang Medical University (Henan, China). The use of MSCs has been approved by the Ethics Committee of Xinxiang Medical University.

\section{PU/Fibrin Vascular Scaffold Preparation}

Until then, our research group has done a lot of work similar to the preparation of PU/fibrin vascular scaffolds using electrospinning technology. ${ }^{14-16}$ In short, we dissolved $15 \%$ wt of PU in a tetrahydrofuran (THF)/dimethylformamide (DMF) solution $(3: 1 \mathrm{v} / \mathrm{v})$ and put it in stirring instrument for 24 hours to prepare PU solution. Fibrin with a concentration of $10 \% \mathrm{wt}$ was dissolved in $98 \%$ formic acid solution and stirred for 4 hours to obtain fibrin solution. We mixed PU solution with fibrin solution to prepare homogeneous mass ratio concentration of PU/fibrin (0:100), PU/ fibrin (5:95), PU/fibrin (15:85) and PU/fibrin (25:75) blend solution, respectively. After that, we put the PU/fibrin solution into $10 \mathrm{~mL}$ medical syringe and fixed syringe on the propulsion pump. Then, the parameters of the electrospinning apparatus (FM1012, Dalian, China) were set as: spinning voltage $(18 \mathrm{kV})$, receiving distance $(13 \mathrm{~cm})$, propulsion speed $(0.8 \mathrm{~mL} / \mathrm{h})$ and humidity $(40 \%)$. Under the action of high voltage electric field, the spinning solution solidified and converged with each other to form PU/fibrin fiber. The prepared electrospun materials were ventilated and dried at room temperature for 5 days to evaporate the residual formic acid solvent and stored it in a clean sealed bag.

\section{Observed of PU/Fibrin Scaffolds by SEM}

We used a scanning electron microscope (SEM) (Hitachi Model SU-8010, Japan) to observe the micromorphology of the PU/fibrin (15:85) vascular scaffold. The tested sample was cut into $1.0 \mathrm{~mm}$ (width) $\times 1.0 \mathrm{~mm}$ (length), and the steps of fixation, dehydration and drying were performed, respectively. Then, it was sputtered with gold for 30s, and pictures were collected at an acceleration voltage of $10 \mathrm{kV}$. To evaluate the diameter distribution of the vascular scaffold fibers, we randomly selected 50 nanofibers from the images and measured the fiber diameter by Image $J$ software $(n=3)$.

\section{Evaluation of Mechanical Properties}

\section{Tensile Properties Test}

The vascular scaffolds were pruned as $1.0 \mathrm{~cm}$ (length) $\times$ $0.5 \mathrm{~cm}$ (width). We used electronic universal material testing equipment (Mitsubishi, Japan) to test the samples. After the sample was fixed on the instrument, the load cell was $50 \mathrm{~N}$ and the crosshead speed was $8 \mathrm{~mm} / \mathrm{min}$ until the test sample fractured. The elastic strength, modulus of elasticity and elongation at break were obtained by the stress-strain curve. Hereon, it was important to note that we performed three times measurement on four different samples and calculated the mean and standard deviation (SD). Three samples were measured, respectively.

\section{Suture Strength}

To ensure that the vascular scaffold can be well joint to the native artery, the suture strength is an important indicator. In accordance with the standards set by the China Medical Device Industry Association, the four test samples were cut into $1.5 \mathrm{~cm} \times 2.0 \mathrm{~cm}$, and $4-0$ polydioxanone suture was used to firmly sew it to the end of the universal material testing instrument. The other end of the instrument was the test clip and checked that the two ends were not loose. Then, the test started with elongation rate as $6 \mathrm{~mm} / \mathrm{min}$ until the tested sample was destroyed. The maximum load that the scaffold material borne before tear-off was recorded. Three samples were measured, respectively.

\section{Burst Strength}

Before performing the burst strength test operation, each tubular vascular scaffold material (3cm in length) was soaked with physiological saline for 30 minutes $(n=3)$. Then, the test sample was secured on the instrument by 4-0 non-absorbable silk sutures and connected with a digital pressure gauge. The other end was closed and physiological saline was gradually added into the lumen of the vascular scaffold until the sample ruptured. Furthermore, the maximum pressure value before the rupture of the vascular scaffold was recorded.

\section{Water Contact Angle Detection}

In order to evaluate the hydrophilicity of PU/fibrin vascular scaffolds, we used the contact angle instrument (Fangrui, China) to detect. Each sample was prepared into the same shape and placed on the coverslips. We dropped $0.03 \mathrm{~mL}$ of deionized water on different scaffolds fiber membranes, and then recorded the angle value at the 5 seconds after the water droplets contacted the scaffold fiber membrane. Each sample was measured three times in succession and the average value was calculated.

\section{Analysis of Blood-Scaffold Interaction Experiment of Platelet Adhesion of the Scaffold}

Fresh blood was extracted from the tail vein of SD rats and was mixed with sodium citrate in a certain proportion. 
Then, the supernatant obtained after centrifugation at 112 $g$ for 8 minutes was platelet-rich plasma (PRP). To obtain platelet-poor plasma (PPP), quantitative PRP was centrifuged at $1006 \mathrm{~g}$ for 15 minutes. We put the prepared PPP and PRP in a certain proportion on the sample and soaked it for 2 hours. The sample was washed with PBS buffer three times to remove impurities on the sample surface, and then was fixed with $2.5 \%$ glutaraldehyde for 4 hours. Then, the gradient alcohol $(100 \%, 90 \%, 80 \%, 70 \%, 60 \%$ and $50 \%$ ) was used for dehydration successively. The samples were dried in a vacuum drying oven, and observed by SEM. Three samples were measured, respectively.

\section{Hemolysis Test}

To obtain precipitated red blood cells, we centrifuged the fresh blood with a centrifuge (Thermo, USA) at $447 \mathrm{~g}$ for 6 minutes and the supernatant was removed. Then, it was thoroughly mixed with physiological saline to prepare an appropriate concentration of red blood cell suspension. The sterilized PU/fibrin vascular scaffolds were cut into $5 \mathrm{~cm}$ (length) $\times 5 \mathrm{~cm}$ (width) shape each. Simultaneously, red blood cell suspension $(200 \mu \mathrm{L})$ was added to each sample. Moreover, the positive group was sterile distilled water $(5 \mathrm{~mL})$, and the negative group was normal saline $(5 \mathrm{~mL})$. The four tested samples were incubated for 2 hour and centrifuged at $447 \mathrm{~g}$ for 20 minutes. Excess liquid was removed and placed in the well plate. The enzyme-labeled instrument (Thermo Fisher, USA) was used for detection at $540 \mathrm{~nm}$. The hemolysis rate (HR) was calculated according to the following formula.

$$
\operatorname{HR}(\%)=\frac{\mathrm{At}-\mathrm{Anc}}{\mathrm{Apc}-\mathrm{Anc}} \times 100 \%
$$

The absorbance of the sample was represented by At; Anc represented the absorbance of the negative control and Apc represented the absorbance of the positive control, respectively. Each sample was measured three times to obtain the HR value.

\section{Dynamic Coagulation Assay}

We prepared four different test samples of equal size and washed them with PBS. The processed samples were placed in beaker respectively. Then, we separately mixed anticoagulated blood $(1 \mathrm{~mL})$ and $\mathrm{CaCl}_{2}(0.3 \mathrm{~mL})$ with the vascular material, and placed them in a $37^{\circ} \mathrm{C}$ pot for incubation10, 20, 30, 40 and 50 minutes, respectively. Then, $25 \mathrm{~mL}$ deionized water was added and shook at a constant temperature of $37^{\circ} \mathrm{C}$ for $5 \mathrm{~min}$, and then the solution was took out for measuring absorbance at a wavelength of $415 \mathrm{~nm}$ (As) with an ultraviolet spectrophotometer (Meipuda, China). The absorbance of the mixture of $1 \mathrm{~mL}$ blood and $25 \mathrm{~mL}$ deionized water was assumed as a reference value (Ar). The blood clotting index (BCI) calculation formula was as follows $(n=3)$.

$$
\mathrm{BCI}=\frac{\mathrm{As}}{\mathrm{Ar}} \times 100 \%
$$

\section{Antithrombin Trial}

In order to explore the influence of vascular scaffolds on the antithrombin, we cut the four samples to a uniform size and sterilized them with ultraviolet light for 2 hours. The test samples and rat blood plasma $(400 \mu \mathrm{L})$ were added to the centrifuge tube in turn, and incubated in a $37^{\circ} \mathrm{C}$ water bath for 2 hours. We took this group without vascular scaffold material as a control group. Automatic coagulation instrument (SYSMEX CA-7000, Japan) was used to detect the activated partial thromboplastin time (APTT) and thromboplastin time (TT) of four samples. The tested data were obtained through three times measurement.

\section{Investigation on the Degradation of Vascular Scaffolds in vitro}

The four different vascular scaffolds were cut into $2.0 \mathrm{~cm}$ (length) $\times 2.0 \mathrm{~cm}$ (width), and then submerged in sterile PBS solution ( $\mathrm{pH}$ 7.4) and lipase solution $(\mathrm{pH} \mathrm{7.4,1} \mathrm{mg}$ $\mathrm{mL}^{-1}$ ) respectively, and placed in the oscillator (THZ-100, China) at $37^{\circ} \mathrm{C}$ for incubation. The lipase solution should be replaced weekly. In addition, the weight of vascular scaffolds were measured at 1 week, 2 weeks, 3 weeks and 4 weeks to calculate the mass loss rate $(n=3)$. We observed the microscopic morphological changes of the vascular scaffolds degradation under SEM and collected images.

\section{Analysis of Cell-Scaffold Interaction Live Cell Staining Test}

We sterilized the prepared four kinds of electrospun scaffold materials under ultraviolet light for 3 hours and placed them in 48-well plates, respectively. Then, DMEM $(100 \mu \mathrm{L})$ was added to each well and incubated in a $5 \% \mathrm{CO}_{2}$ incubator at $37^{\circ} \mathrm{C}$ for 5 hours. MSCs were seeded with a density of $1.0 \times 10^{4}$ cells/well. The medium in the well plate was changed every 2 days. The morphology of MSCs was observed by living cell staining kit at 3 and 5 days, respectively. We removed the old medium and washed it with PBS 3 times. Then, a certain concentration of calcein $(2 \mu \mathrm{L})$ was added into each well under the dark light environment, and 
incubated for 20 minutes. After the samples were washed with PBS for three times, we used fluorescence microscope to take photos of different scaffolds.

\section{Proliferation of MSCs on Scaffolds}

We used the CCK-8 kit to assess the number of MSCs. In short, we cultured electrospun scaffolds and MSCs in 96well plates at 1, 3, and 5 days. Then, the old medium was discarded and the samples were rinsed with PBS for three times. DMEM $(400 \mu \mathrm{L}$ in total) was added, and CCK-8 $(30 \mu \mathrm{L})$ was added to each well in the dark environment, and then incubation at $37^{\circ} \mathrm{C}$ for 2 hours. The absorbance was measured at $540 \mathrm{~nm}$ by a multifunctional enzyme analyzer (Thermo Fisher, USA) at three different time points. Three samples were measured, respectively.

\section{Investigation on the Gene Expression by Quantitative RT-PCR}

In order to obtain RNA, we co-cultivated four different PU/ fibrin scaffold materials with MSCs for 6 days, and obtained total RNA according to the instructions. All RNA was synthesized into cDNA by HieffTM First Strand cDNA Synthesis Super Mix reagent (Shanghai Yiji Industrial Co., Ltd., China). Then, HieffTM qPCR SYBR ${ }^{\circledR}$ Green Master Mix (Shanghai Yiji Industrial Co., Ltd., China) was used for quantitative fluorescence PCR. In addition, the primer sequence of collagen was forward primer: 5'-TGACC CTGGTTCATGGGATG-3', reverse primer: 5'-TTCCCCGT GGCTGATATTCC-3'. The primer sequence of survivin was forward primer: 5'-CCTACGGAAGAAAGCCAGACA-3', reverse primer: 5'- GCCCAGGTATGTCAGGATGC-3'. The primer sequence of vimentin was forward primer:5'CTAGCCGCAGCCTCTATTCC-3', reverse primer: 5'-GT CCACCGAGTCTTGAAGCA-3'. GAPDH (forward primer: 5'-ACCACAGTCCATGCCATCAC-3'; reverse primer: 5'-TCCCCACCCTGTTGCTGTA-3') was used in internal control group. The result values of all genes were obtained by the $2^{-\Delta \Delta C T}$ method. Three samples were measured, respectively.

\section{Performance Evaluation of Vascular Grafts in vivo \\ Graft Transplantation in vivo}

All animal experiments were approved by the Animal Ethics Committee of Xinxiang Medical University (Henan, China). All animal surgical experiments followed the guidelines of the National Institutes of Health for the care and use of animal experiments. Adult male SD rats $(n=45$, average weight 250g) were from Xinxiang Medical University. The PU graft $(n=15)$ were used as the control group, while PU/ fibrin $(15: 85)$ graft $(n=15)$ was the experimental group. We used $70 \%$ ethanol to immerse the vascular graft and rinsed it repeatedly with PBS. We used 5\% chloral hydrate to anesthetize SD rats and removed the hair from the operation area. Then, the iodophor solution was used to routinely sterilize the abdomen of the SD rats. We made a $4 \mathrm{~cm}$ incision longitudinally, and then gradually separated the subcutaneous tissues until it reached the abdominal aorta. To ensure that there were no nerves and blood vessels damaged, we used surgical vascular forceps to block the abdominal aorta and disconnected it. We used 8-0 surgical suture to anastomose the broken ends with the PU/fibrin grafts and reestablished the vascular return channel. The abdominal cavity was closed after no active bleeding was observed. In addition, appropriate antibiotics were applied before and after surgery, and aspirin $15 \mathrm{mg} / \mathrm{kg}$ was took every day. Animal models were sacrificed at 1, 2, and 3 months after transplantation, respectively. Here we need to explain that four SD rats died accidentally after the operation. Survival rate was $91 \%$. We observed the patency of the vascular graft with a stereo microscope (Xingming Optics, China). Moreover, we used $4 \%$ paraformaldehyde to fix the spare samples and stored them in the refrigerator.

\section{Functionality Assessment of Vascular Grafts}

The aortic ring bioassay experiment has been described in our previous work. After the removed vascular explants were cleaned with PBS, they were submerged in buffer solution for 3 hours. Ten percent KCL solution $(45 \mathrm{mM})$ and sodium nitroprusside (SNP, $1.0 \times 10^{-7} \mathrm{~mol} / \mathrm{L}$ ) were added to detect the constriction and relaxation function of the graft, respectively. In addition, we used adrenaline $(\mathrm{AD}, 0.9 \mathrm{mM})$ solution and acetylcholine (Ach, $10 \mu \mathrm{M})$ drugs to investigate the vascular endothelial function of the graft. We used the force sensor recorder (AD Instruments, Australian) to detect and obtained its value.

\section{Biomechanical Test of Grafts in vivo}

In this work, we had measured the mechanical properties of the vascular scaffold in vitro. With reference to the previous method, we evaluated the elastic modulus, breaking strain and maximum stress of different samples by universal material testing instrument (Mitsubishi, Japan). Furthermore, the sample should be measured more than three times continuously, and then the average value and standard deviation were obtained. 
Compliance is to examine the degree of graft response under vascular pulse pressure. After the anesthetized rats were lethargic, it was placed on small animal ultrasound equipment (Vevo3100, Canada) for imaging operation. Then, we obtained the values of diastolic and systolic blood pressure by the animal blood pressure measuring instrument (NIBP200A, USA). Compliance value was obtained by the following formula.

$$
\text { Compliance per } 100 \mathrm{mmHg}(\%)=\frac{\mathrm{Rp} 2-\mathrm{Rp} 1}{R p 2(\mathrm{P} 1-\mathrm{P} 2)} \times 10^{4}
$$

P1 indicated the detected high-pressure value, P2 indicated the low-pressure value ( $\mathrm{mmHg}$ ). $\mathrm{Rp} 1$ and $\mathrm{Rp} 2$ indicated the inner diameter values of high pressure and low pressure, respectively.

\section{Immunofluorescence Staining}

For immunofluorescence staining experiments, we selected PU/fibrin (15:85) group, PU group and native group samples at 3 months for operation. In short, we prepared frozen sections of these samples. Then, $5 \%$ fetal bovine serum and $0.2 \%$ TritonX-100 solution were added to treat these samples. To observe smooth muscle cells (SMCs) in vascular grafts, we stained them with mouse anti- $\alpha$-SMA (1:150) antibody. Simultaneously, the endothelial cells (ECs) in the graft were investigated by rabbit anti-von Willebrand factor (vWF, 1:150). Then, the inflammatory cells in the samples were evaluated by staining with the mouse anti-CD68 antibody (1:250). To clearly identify the nucleus, DAPI $(1: 1000)$ solution was added to all samples for staining. In addition, we analyzed TNF- $\alpha$, IL-6, IL-10 and IL-4 inflammatory factors by ELISA experiments. All fluorescence images were obtained by a fluorescence microscope (Olympus BX53TR, Japan) and calculated the data with ImageJ software.

\section{Histological Analysis}

SD rats were sacrificed at 3 months after transplantation. We rinsed the tested samples with PBS and fixed them with $4 \%$ paraformaldehyde. The vascular grafts were cut into the same size and completely immersed in paraffin before embedding. We took PU grafts, PU/fibrin (15:85) grafts and native artery samples with a thickness of $4 \mu \mathrm{m}$. Then, we strictly followed the instructions of the kit to stain the samples with Safranin O, Verhoeff and Sirius Red, respectively. Through staining experiments, we obtained the distribution of glycosaminoglycan (GAG), elastin and collagen and also quantitatively analyzed their contents. In addition, all the photos were obtained with an upright microscope (NIKON ECLIPSE E100, Japan). Professor Xiaoyu Yang, a pathologist at Xinxiang Medical University, has reviewed the staining slides and score.

\section{Statistical Analysis}

All experiments were established at least 3 parallel samples. The data obtained in the experiment were expressed as mean $\pm \mathrm{SD}$. For statistical analysis, Graphpad Prism 7 and OriginPro2018 software were used for calculation. $\mathrm{P}<0.05$ indicated significant difference in statistics.

\section{Results}

\section{Morphology of Electrospun PU/Fibrin Scaffold}

We could see that fibrin (Figure 1A) and PU/fibrin (Figure 1B) scaffolds were successfully prepared by electrospinning technology. The vascular scaffold had a relatively tight nanofiber membrane structure, and there was no delamination phenomenon. The micromorphology of fibrin and PU/fibrin vascular scaffolds was observed under SEM. Figure $1 \mathrm{C}$ and D showed that the fibers of the fibrin and PU/fibrin vascular scaffolds randomly oriented, and the fiber surface was smooth and without beads. Fifty fibers were randomly selected from the two sample images and the fiber diameter was detected by ImageJ software. The fiber diameter of PU/fibrin was 712 $\pm 23 \mathrm{~nm}$, which was smaller than that of fibrin (Figure 1E-F).

\section{Characterization of Mechanical Properties and Hydrophilicity}

In order to maintain the vascular scaffold enough to resist the impact of blood flow, the mechanical strength is a very important indicator. According to the results in Figure 2A and $\mathrm{C}$, it could be observed that PU/fibrin (25:75) vascular scaffolds had the highest tensile strength and elongation at break, while the pure fibrin vascular scaffolds had the lowest tensile strength and elongation at break. With the PU content in the PU/fibrin vascular scaffold gradually increased, its corresponding mechanical properties also increased, but it was surprisingly found that its modulus of elasticity decreased (Figure 2B). In addition, there were statistical differences in tensile strength, modulus of elasticity and elongation at break between the four PU/fibrin scaffolds.

To investigate the successful application of scaffold in vivo vascular transplantation, the suture strength of vascular scaffold is another key experimental index. It 

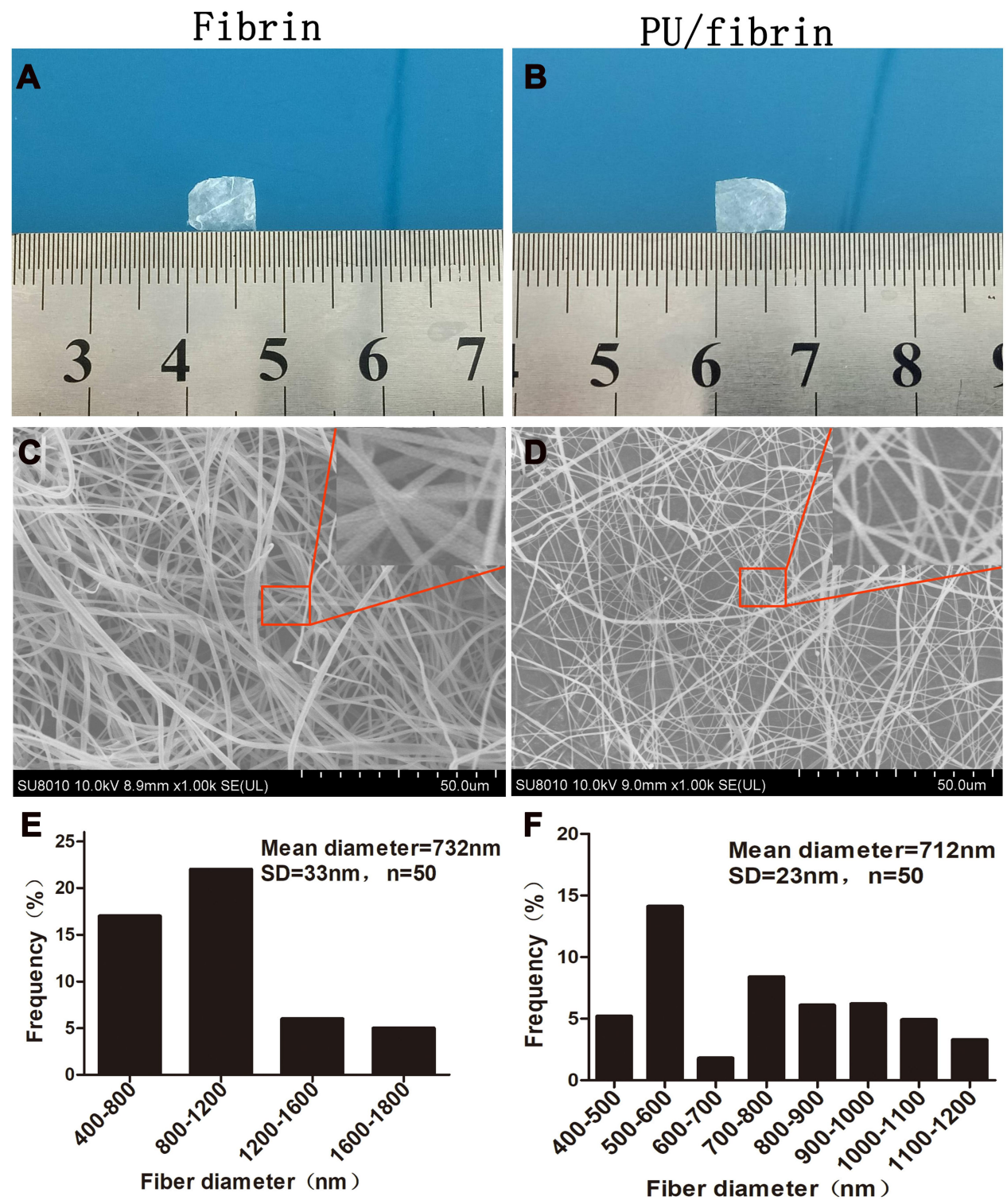

Figure I Morphological observation and fiber diameter analysis of electrospun vascular scaffolds. The macroscopic morphology of fibrin (A) and PU/fibrin (I5:85) (B) scaffolds was collected with the camera. Microstructures of fibrin (C) and PU/fibrin (I5:85) (D) vascular scaffolds were observed by SEM. The fiber diameter distribution of fibrin (E) and PU/fibrin (I5:85) (F) were measured by ImageJ software $(n=3)$.

could be seen from the results in Figure 2D that the suture strength of the PU/fibrin composite vascular scaffold increased with the increase of PU content. The vascular scaffold of PU/fibrin (5:95), PU/fibrin (15:85) and PU/ fibrin (25:75) met the requirement of ideal suture strength for a long time before fracture. Furthermore, there was a significant difference in suture strength between four different mass ratios of PU/fibrin scaffolds.

The burst pressure is also a key indicator for vascular scaffold. The results showed that the PU content in the $\mathrm{PU} /$ fibrin vascular scaffolds increased from $5 \%$ to $25 \%$, and the corresponding burst pressure value also increased from $1420 \pm 123.7 \mathrm{mmHg}$ to $1947 \pm 62.7 \mathrm{mmHg}$. At the same time, the burst pressure values of PU/fibrin (0:100), PU/fibrin (5:95), PU/fibrin (15:85) and PU/fibrin $(25: 75)$ vascular scaffolds were statistically significant $(\mathrm{p}<0.05)$ (Figure 2E).

To evaluate the hydrophilicity of the vascular scaffold, the contact angles of four PU/fibrin scaffolds were tested. The results indicated that the contact angle values of the pure fibrin vascular scaffold was about $22.1^{\circ}$. Moreover, with the increase of PU content, the contact angle values of scaffold gradually increased, and the contact angle value of PU/fibrin (25:75) scaffolds was $49.7^{\circ}$, which 


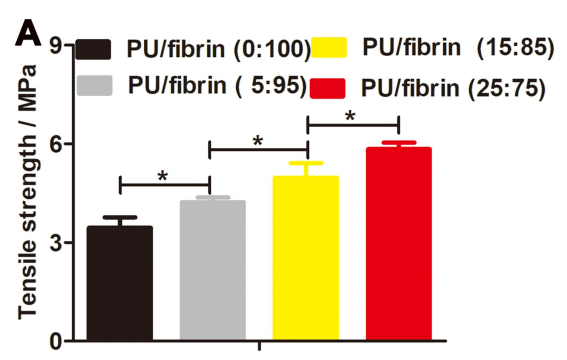

Vascular scaffold

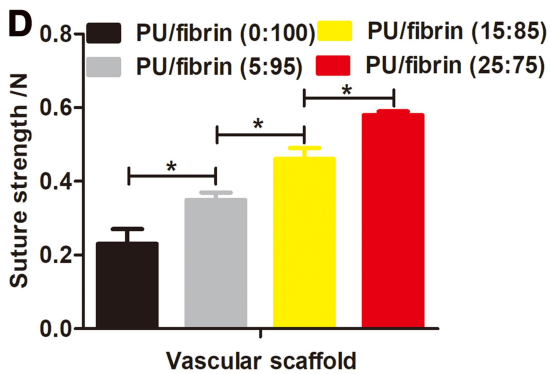

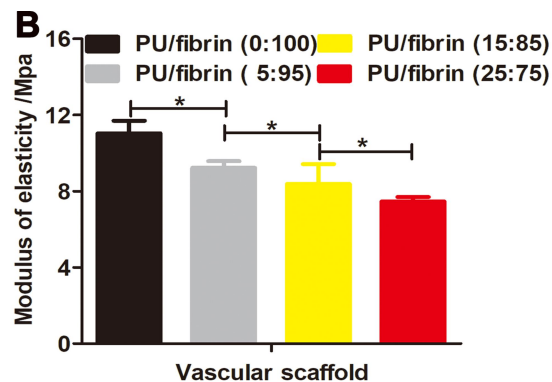

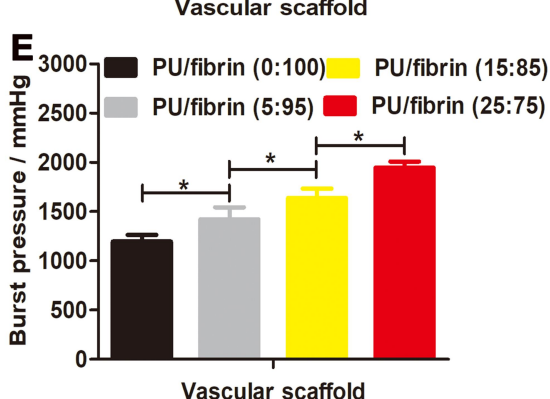

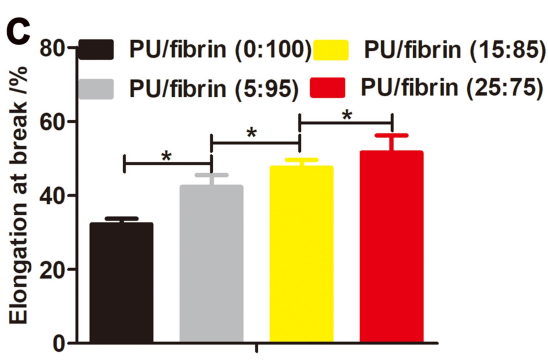

Vascular scaffold

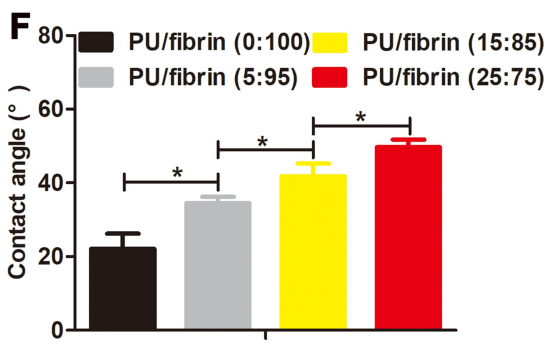

Vascular scaffold

Figure 2 Evaluation of mechanical strength and hydrophilicity of electrospun PU/fibrin vascular scaffolds. The tensile strength (A), modulus of elasticity (B), elongation at break (C), suture strength (D) and burst pressure $(\mathbf{E})$ were compared between four different ratio of $\mathrm{PU} / \mathrm{fib}$ rin scaffolds ( $\mathrm{n}=3$ ). The water contact angle values of the vascular scaffolds $(\mathbf{F})$ were measured $(n=3)$. $\star$ indicated $P<0.05$.

indicated that PU could effectively reduce the hydrophilicity of the composite scaffold (Figure 2F).

\section{Investigation of Hemocompatibility of Electrospun Scaffolds in vitro}

SEM showed that there were few platelets on the surface of scaffolds in the two groups, which showed that the two scaffolds had good antiplatelet adhesion (Figure 3A and B). Figure $3 \mathrm{C}$ shows the hemolysis value of scaffold material, and the hemolysis values of different proportions of electrospun scaffolds were not more than $2 \%$, lower than $5 \%$ of the international standards. The BCI value of PU/fibrin (0:100), PU/fibrin (5:95), PU/fibrin (15:85) and PU/fibrin (25:75) scaffolds at $10 \mathrm{~min}$ were $75.4 \pm 2.19 \%, 72.1 \pm 3.3 \%, 61.4 \pm 3.6 \%$ and 57.2 $\pm 2.1 \%$, respectively, without difference in statistics in different groups (Figure 3D). It also can be observed that the $\mathrm{BCI}$ value decreased with the increase of PU content. The results demonstrated that TT and APTT of different scaffolds were about 16s and $24 \mathrm{~s}$ respectively (Figure $3 \mathrm{E}$ and F). For the four PU/fibrin hybrid scaffolds, TT values were in the normal value range (15-18s). In detail, the APTT value did not change with the change of PU content.

\section{Degradation in vitro of Vascular Scaffolds}

To investigate the degradation performance of vascular scaffolds in vitro, four different electrospun scaffolds in phosphate-buffered saline (PBS) and lipase solution were tested, respectively. With the increase of degradation time, the mass of all vascular scaffolds continued to decrease. At the same time, the mass loss rate of all vascular scaffolds in enzymatic hydrolysis was significantly higher than that of corresponding group in PBS (Figure 4A and B). In addition, we found that the mass loss of the four different vascular scaffolds in hydrolytic degradation was relatively low (Figures 4A and 4C1-C4). Compared with PU/fibrin (5:95), PU/fibrin (15:85) and PU/fibrin (25:75) scaffolds, PU/fibrin (0:100) vascular scaffolds had the fastest degradation rate in enzyme degradation. After incubation in lipase solution for 4 weeks, the mass loss rate of PU/fibrin (0:100), PU/fibrin (5:95), PU/fibrin (15:85) and PU/fibrin $(25: 75)$ vascular scaffolds were $23.4 \pm 1.11 \%, 18.6 \pm$ $1.35 \%, 16.2 \pm 0.55 \%$ and $13.7 \pm 0.85 \%$, respectively (Figure 4B). We used SEM to observe the morphology of different vascular scaffolds in hydrolytic degradation. The results showed that the four scaffolds still maintained a certain appearance, and the degree of degradation was very low (Figure 4C1-C4). However, it was interesting that the four vascular scaffolds showed significant degradation behavior in enzyme degradation. In particular, PU/ fibrin $(0: 100)$ scaffolds had a large number of broken fibers and fiber adhesion. Compared with the other three vascular scaffolds, PU/fibrin (0:100) vascular scaffold degraded the most deeply (Figure 4D1-D4). 

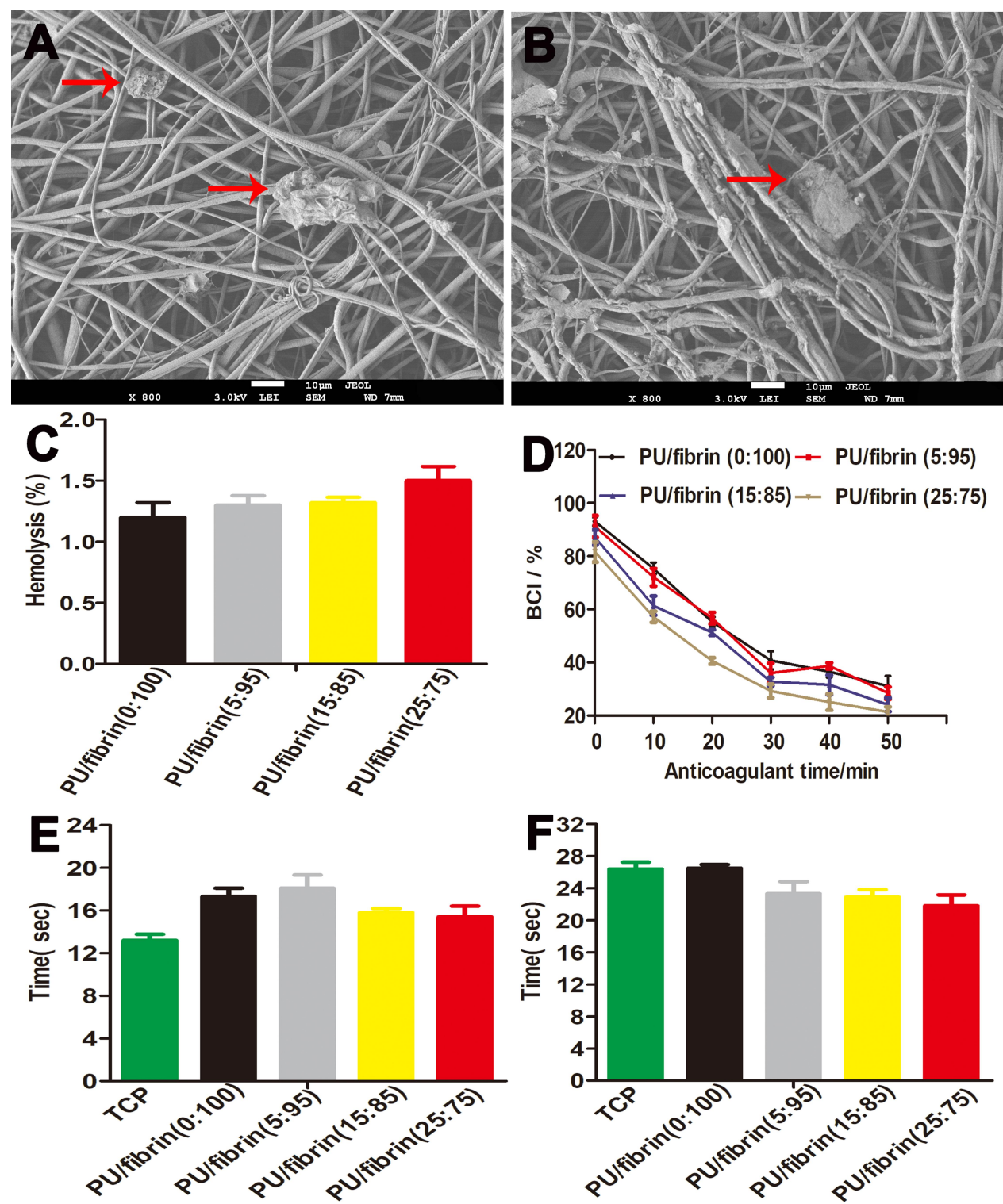

Figure 3 The hemocompatibility of electrospun vascular scaffolds. SEM images of platelet adhesion on PU/fibrin (A) and fibrin (B) vascular scaffolds surface. The red arrows in the SEM images indicated adherent platelets. The hemolysis percentage $(\mathbf{C})$, dynamic coagulation time (D), TT (E) and APTT (F) were measured $(n=3)$.

\section{Proliferation Behavior of MSCs and Gene Expression of Scaffolds Extract}

We used live cells staining methods to investigate the viability of MSCs on four electrospun scaffolds (Figure 5A). The MSCs were cultured on scaffolds for 3 and 5 days, respectively. The results showed that the number of living cells on PU/fibrin (25:75) scaffolds was significantly lower than on the other three scaffolds. At the same time, the number of living cells between PU/fibrin (0:100), PU/fibrin (5:95), PU/fibrin (15:85) scaffolds was almost the same. To evaluate the proliferation of MSCs quantitatively on different vascular scaffolds, CCK- 8 reagent was used to detect (Figure 5B). The results showed that the cell proliferation on different vascular scaffolds had no statistically significant difference at 1 day. However, PU/fibrin (25:75) vascular scaffolds were significantly different from the other three PU/fibrin scaffolds at 3 and 5 days. Furthermore, the higher the absorbance value at $450 \mathrm{~nm}$ indicated the larger number of MSCs in the scaffolds. We also found that the number of cells on the vascular scaffolds increased with coculture time prolonged. The gene expression of MSCs cocultured with four different PU/fibrin electrospun scaffolds extract for 6 days (Figure 5C) was researched. The results showed that the expression of collagen in PU/fibrin $(25: 75)$ group was significantly lower than that in other PU/fibrin groups. Meanwhile, the expression of survivin gene had 

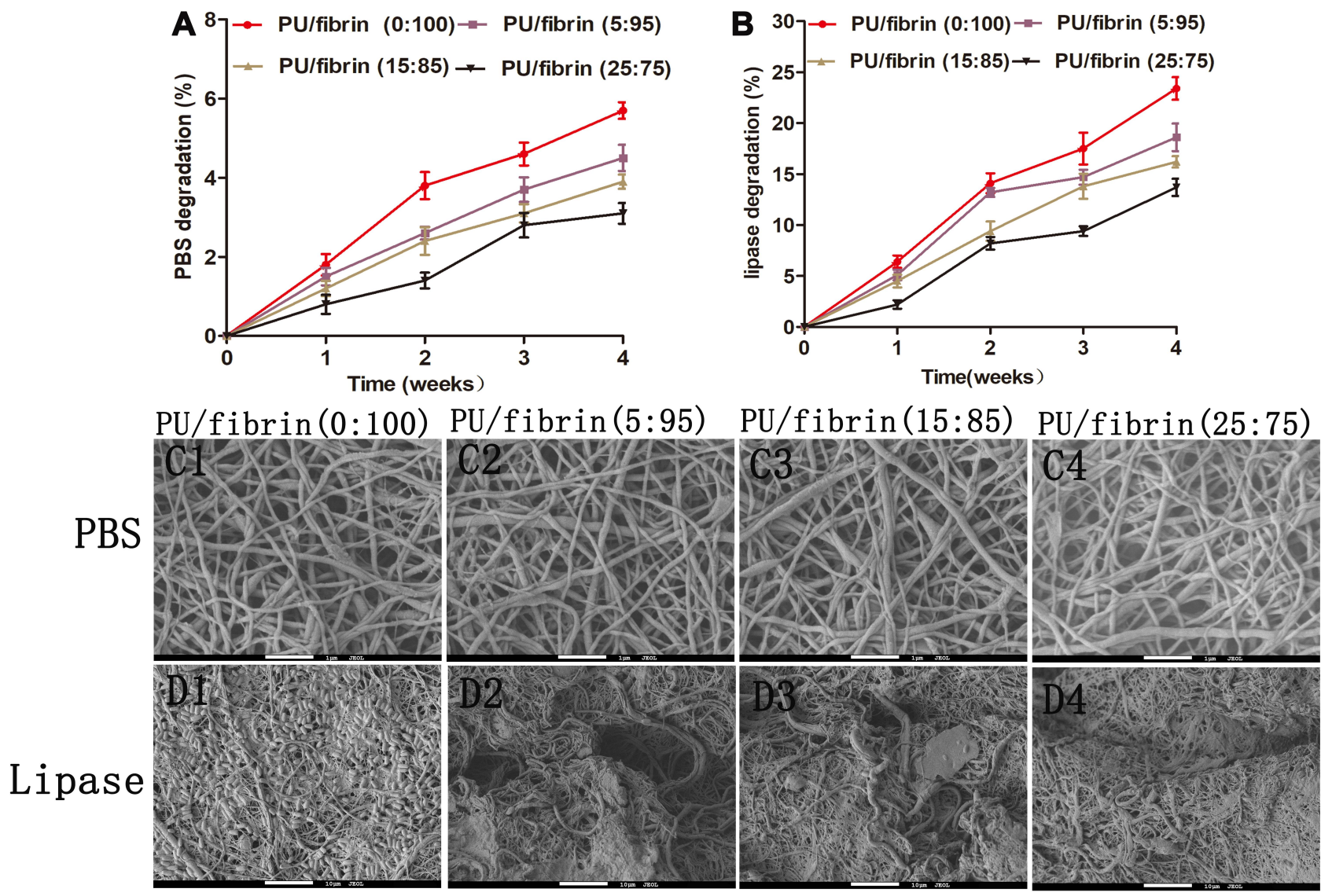

Figure 4 Analysis of degradation in vitro of the PU/fibrin vascular scaffolds. The PBS degradation (A) and lipase degradation (B) of vascular scaffolds were evaluated. Morphological observation of different vascular scaffolds after treatment with PBS (CI-C4) and lipase solution (DI-D4) for 4 weeks by SEM.

statistical difference in PU/fibrin (0:100) group and PU/ fibrin (5:95) group, PU/fibrin (15:85) group and PU/fibrin (25:75) group, respectively. The gene expression of vimentin in the PU/fibrin (25:75) group was statistically significant between the other PU/fibrin groups.

\section{The Patency, Functionality and Mechanical Properties of Vascular Grafts in vivo}

We found from Figure 6A that the PU/fibrin (15:85) vascular graft was similar to the native artery at 3 months, but the graft at 1 month and 2 months was significantly different from the native artery. Meantime, there was no obvious bleeding at the anastomotic site, and the formation of vascularization on the surface of the graft. Interestingly, the neoartery of the PU/fibrin (15:85) vascular grafts was difficult to distinguish from the native arteries after being treated with PBS (Figure 6B). The vascular grafts were observed by stereomicroscope at three time points. The thickness of the graft lumen gradually changed with the time of implantation increased, and then some neotissues were generated on the surface of the graft lumen (Figure 6C). Furthermore, we evaluated four performance indicators of the vascular grafts. The results showed that the values of patency and viability in PU/fibrin grafts were much higher than those in PU grafts (Figure 6D). It was interesting that the values of acute thrombosis and occlusion in PU/fibrin grafts were lower than those in PU grafts (Figure 6E). The aortic ring bioassay was used to assess the functionality of vascular grafts at 3 months. The results showed that under the action of vasomotor agonists (KCL and $\mathrm{AD}$ ), the constriction of PU/fibrin vascular graft was greater than that of PU vascular graft, but it was lower than that of native artery (Figure 6F). Under the action of vasodilators (Ach and SNP), the relaxation of PU/ fibrin neoartery was similar to that of native artery, but it was still much lower than that of PU vascular graft (Figure 6G). The elastic modulus, breaking strain and maximum stress of PU/fibrin vascular grafts at 3 months were significantly lower than those of PU vascular grafts. However, the mechanical strength of PU/fibrin grafts was closed to that of native arteries. The PU group was significantly different from the PU/fibrin group and the native artery group (Figure 6H-J). 


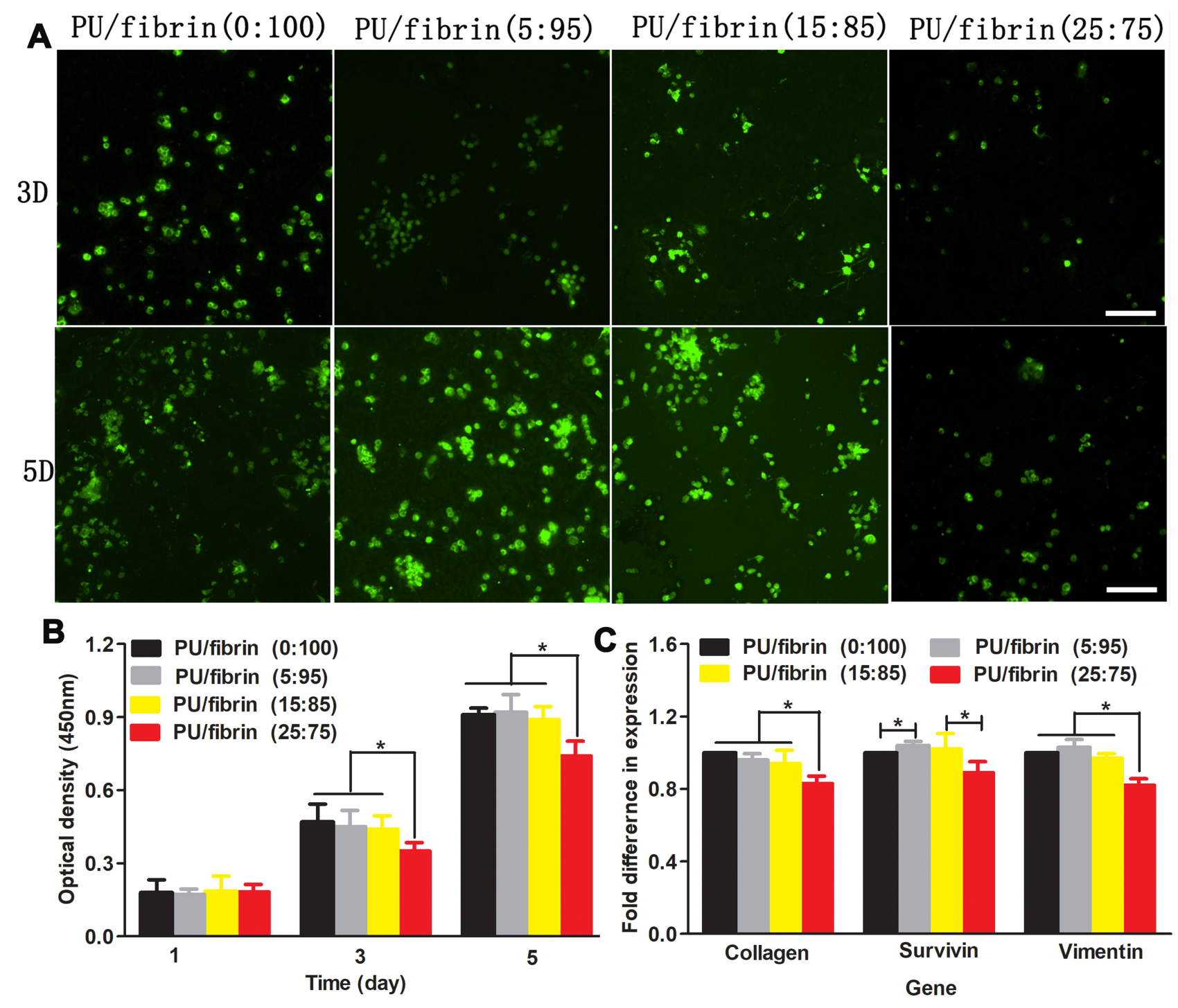

Figure 5 Analysis of proliferation and gene expression of MSCs under different vascular scaffolds. MSCs adhered and proliferated on the vascular scaffolds and live cells were stained with green color (A). The optical density values of cells cultivated on the vascular scaffolds after I, 3 and 5 days (B). Gene expression of cells co-cultivated with scaffold extract for 6 days (C). Scale bar: $500 \mu \mathrm{m}$. $\star$ indicated $\mathrm{p}<0.05$.

Moreover, we also tested the compliance of PU/fibrin vascular grafts at 1 month, 2 months and 3 months, respectively. The results showed that the compliance of PU/fibrin grafts significantly increased with the prolongation of implantation time, until the value of the graft at 3 months was similar to that of the native artery (Figure 6K).

\section{Cell Infiltration and Immunoreaction of Vascular Grafts in vivo}

The immunofluorescence experiment results showed that there was the presence of cell infiltration and neo-tissue formation in the PU/fibrin (15:85) grafts wall at 3 months. At the same time, the number of $\alpha$-SMA positive cells in the PU/fibrin (15:85) group and the native group was significantly higher than that in the PU group. Through immunofluorescence images, we analyzed that there was no significant difference in the expression of $\alpha$-SMA between the PU/fibrin (15:85) group and the native group (Figure 7A and $\mathrm{B})$. To confirm the formation of endothelialization in the graft lumen, we detected it by VWF factor staining. The results showed that compared with PU grafts, PU/fibrin (15:85) grafts and the native artery had obviously continuous endothelial layer. Interestingly, the quantitative analysis of vWF expression in the fluorescence area also further showed that vWF cells in PU vascular grafts were much lower than those in PU/fibrin (15:85) grafts (Figure 7C). Furthermore, in order to investigate the distribution of macrophages in vascular grafts, we used CD68 markers for analysis. At 3 months 

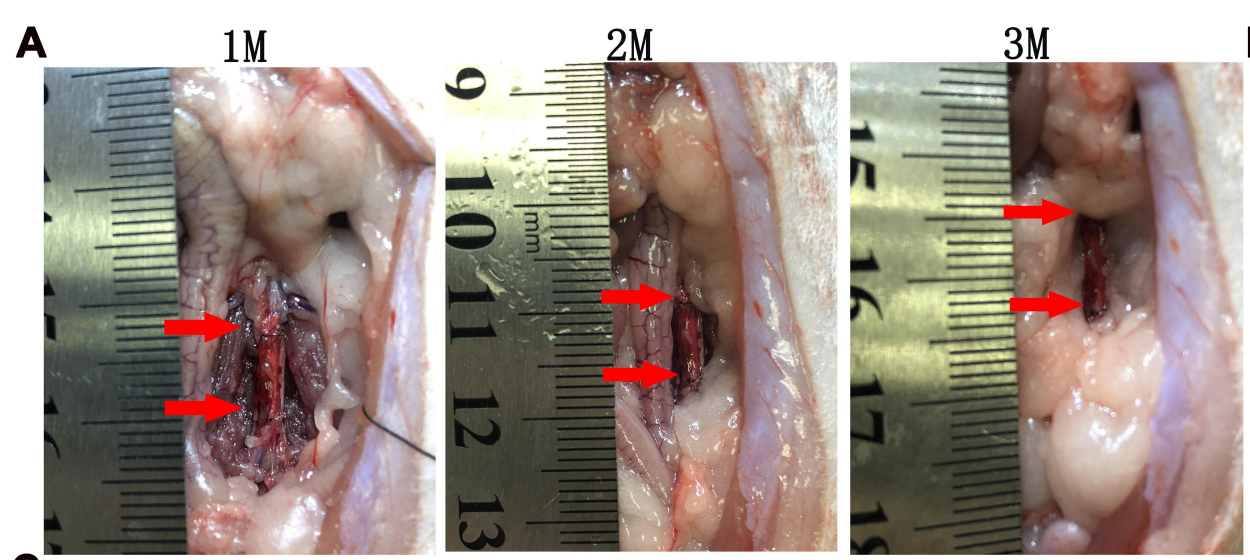

B $\quad 3 \mathrm{M}$
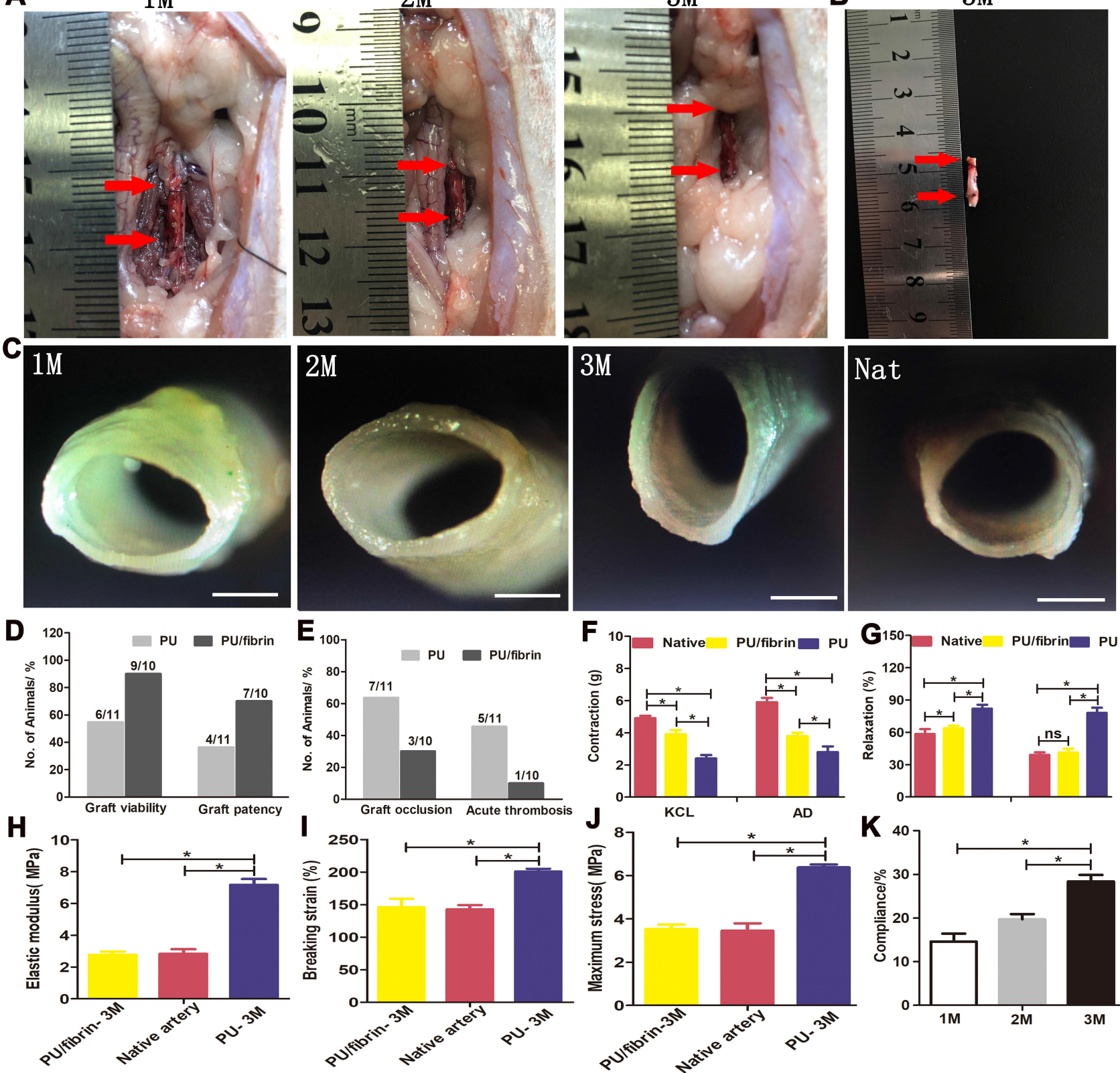

Figure 6 Morphology, patency, functionality, and mechanical properties of vascular grafts. PU/fibrin (I5:85) vascular grafts were transplanted into the abdominal aorta of SD rats for I, 2, and 3 months and the red arrows represented the distal and proximal locations of the graft (A). The PU/fibrin (I5:85) neoarteries at 3 months were washed with PBS solution and the red arrows represented the distal and proximal locations of the graft (B). The cross-section images of the PU/fibrin (I5:85) graft observed by stereo microscope (C). Quantitative data were used to analyze the viability and patency performance of vascular grafts in vivo (D and $\mathbf{E})$. Constriction response of different samples under the action of $K C L$ and AD $(\mathbf{F})$. Ach and SNP were used to evaluate the relaxation response of different vascular grafts $(\mathbf{G})$ ( $n=3)$. The elastic modulus $(\mathbf{H})$, breaking strain $(\mathbf{I})$ and maximum stress $(\mathbf{J})$ of different vascular grafts were evaluated, respectively $(\mathrm{n}=3)$. The compliance of PU/fibrin vascular grafts was measured at $\mathbf{I}, 2$ and 3 months $(\mathbf{K})$. Scale bar: I mm. $\star$ indicated $P<0.05$.

of implantation, there were few CD68 cells in PU/fibrin vascular grafts, which were similar to those in native arteries. However, the number of CD68 positive cells in PU vascular grafts was much more than those in the two other groups. Simultaneously, the expression of CD68 in the PU/fibrin (15:85) group was not statistically different from the native arteries, but the PU group had significant statistical significance with the other two groups (Figure 7A-D).

The pro-inflammatory and anti-inflammatory cytokines in macrophages play important role in the reconstruction and remodeling of artificial blood vessels. Thus, some inflammatory factors were evaluated by ELISA. The results showed that 


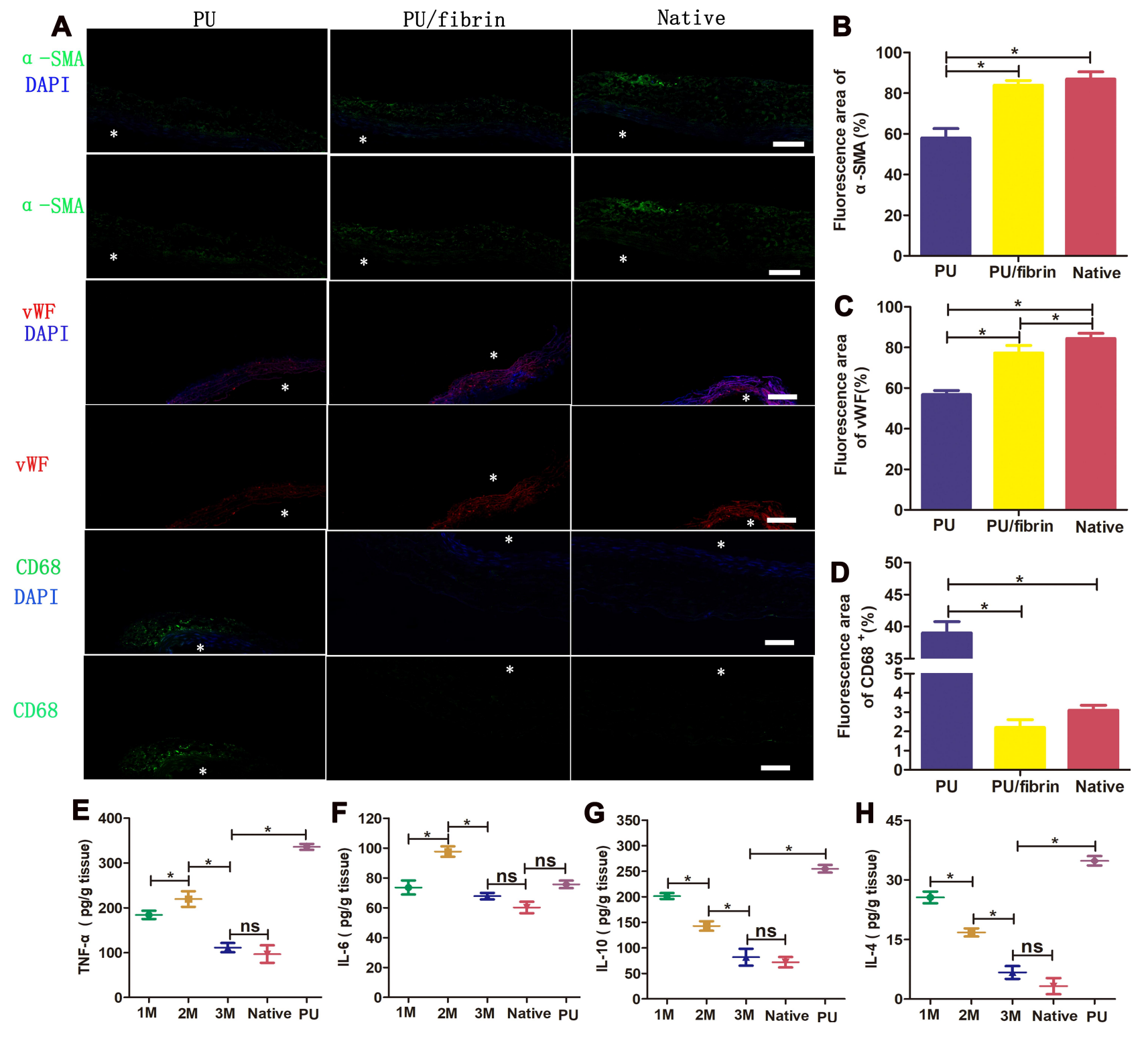

Figure 7 Regeneration of VSMCs and ECs and immunoreaction in the PU/fibrin (I5:85) vascular grafts. Immunofluorescence images of the samples at 3 months: $\alpha$-SMA (green) represented SMCs, vWF (red) represented ECs, CD68 (green) represented macrophages and DAPI (blue) represented cell nuclei, respectively $(\mathbf{A}) .{ }^{*}$ (white color) indicated the lumen of the vascular graft. Scale bar: $100 \mu \mathrm{m}$. Quantitative analysis of $\alpha$-SMA (B), vWF (C) and CD68 (D) expression in vascular grafts, respectively ( $=3$ ). The inflammatory cytokines TNF- $\alpha(\mathbf{E})$, IL-6 $(\mathbf{F})$, IL-I0 $(\mathbf{G})$ and IL-4 $(\mathbf{H})$ were detected by ELISA test $(n=3)$. $\star$ (black color) indicated P $<0.05$. ns indicated no significance.

the secretion of pro-inflammatory cytokines TNF- $\alpha$ and IL-6 increased in PU/fibrin vascular grafts from 1 month, and reached the highest level at 2 months $(219.6 \pm 17.4 \mathrm{pg} / \mathrm{g}$ and $97.7 \pm 3.51 \mathrm{pg} / \mathrm{g}$, respectively). However, the secretion of TNF$\alpha$ and IL- 6 both decreased at 3 months, and finally the secretion levels were similar to that of native arteries (Figure 7E and F). In addition, it was interesting that the secretion of IL-4 and IL-10 in anti-inflammatory cytokines gradually decreased with the increase of implantation times. Importantly, the secretion of TNF- $\alpha$, IL-4 and IL-10 in PU vascular grafts showed high levels of expression at 3 months, but IL-6 was not included.

\section{ECM Deposition in PU/Fibrin Vascular Graft}

As the implantation time increased, changes in ECM deposition (GAG, elastin and collagen) indicated the remodeling of vascular grafts. To investigate the composition of ECM in neoartery, histological staining analysis of glycosaminoglycan (GAG), elastin and collagen were performed at 3 months, respectively. The distribution of GAG in vascular grafts was investigated by safranin $\mathrm{O}$ staining. At 3 months after transplantation, the GAG in the PU/fibrin grafts was neatly arranged in internal layer of the vascular 
grafts and was very similar to the native arteries, but the GAG in the PU grafts was not obvious (Figure $8 \mathrm{~A}-\mathrm{C}$ ). The presence of elastin in vascular grafts was evaluated by using Verhoeff staining. The neointima of the PU/fibrin graft showed elastin deposition, which was similar to native arteries. However, compared to PU/fibrin vascular grafts, the elastin fibers distribution in PU vascular grafts were relatively sparse (Figure $8 \mathrm{E}-\mathrm{G}$ ). The Sirius red staining experiment results indicated that the fibrillar collagen in PU/fibrin vascular grafts was arranged in a circumferential orientation. Moreover, it was surprisingly found that the compactness of PU/fibrin vascular grafts was slightly lower than that of native arteries, but PU vascular grafts were the lowest (Figure 8I-K). According to the quantitative analysis of the ECM components, the results showed that the GAG and elastin in the PU group and PU/fibrin group were lower than those in the native arteries (Figure 8D-H). Interestingly, the collagen content in the PU/fibrin grafts and native arteries was similar, but the collagen content in PU grafts was significantly lower than that in the PU/ fibrin grafts (Figure 8L).

\section{Discussion}

Recent studies have shown that the construction of an ideal small-diameter tissue engineering vascular scaffold material should depend on biocompatibility, nonimmunogenicity, mechanical properties, biodegradability, and non-thrombogenicity. ${ }^{17}$ Simultaneously, the vascular scaffold should also be capable of inducing vascularization, improving microvascular function, and then regenerating functionality similar to native arteries. ${ }^{18,19}$ In addition, researchers have confirmed that materials such as fibrin and PU were suitable candidates for vascular materials, which were widely used in small-diameter tissue engineering vascular field. ${ }^{20,21}$ In our experiments, we successfully used PU and fibrin to prepare a hybrid vascular scaffold, and discussed the mechanical properties, blood compatibility, cell compatibility and degradation performance of PU/fibrin scaffold with different mass ratios. In addition, we also found that PU/fibrin (15:85) can be used as an ideal tubular vascular graft, and evaluated the vascular graft functionality in vivo. The results showed that the surface of the PU/fibrin scaffold were

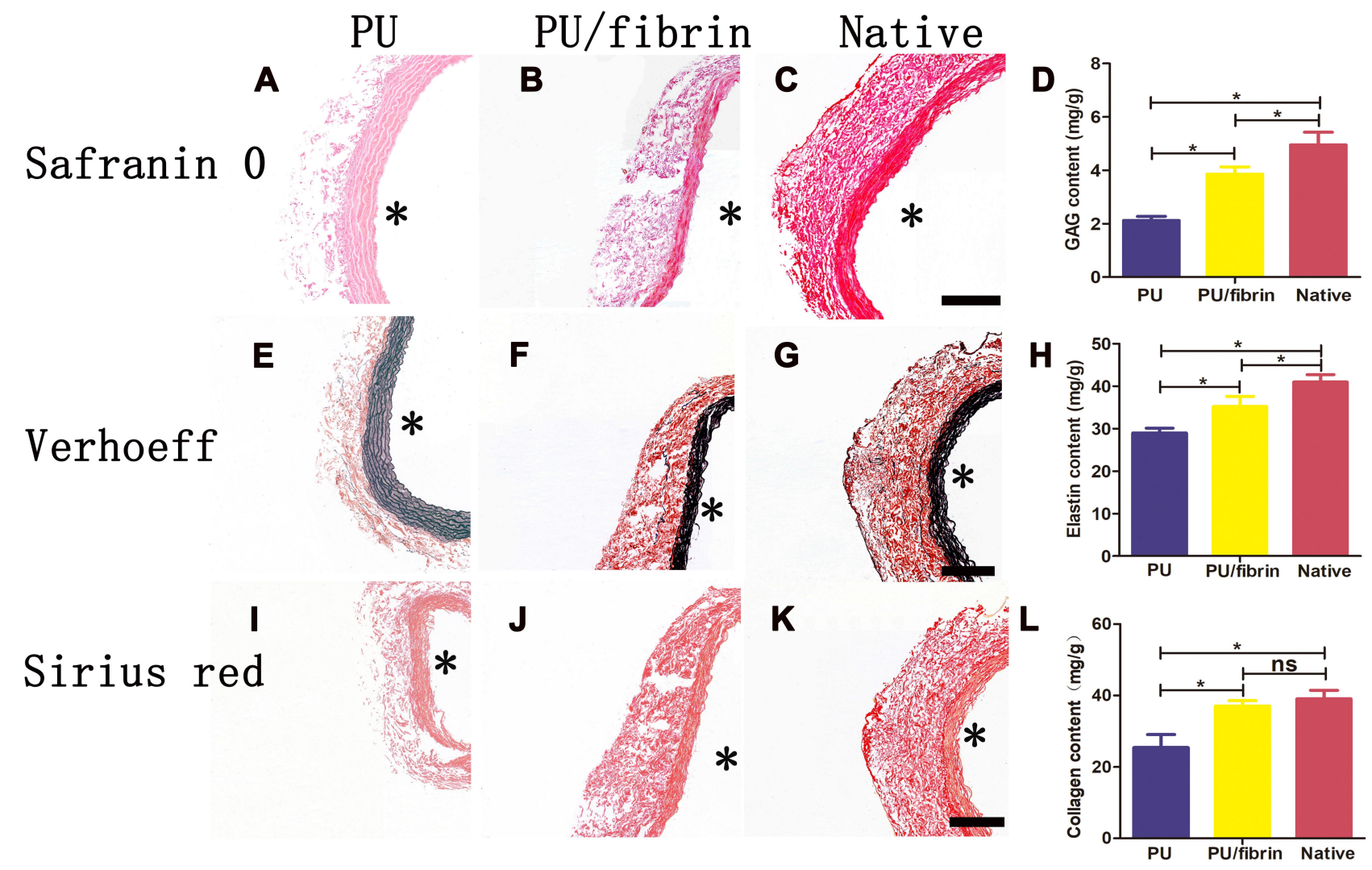

Figure 8 Histological characteristics of ECM changes in PU/fibrin (15:85) vascular graft at 3 months. The samples were stained with Safranin O staining reagent for glycosaminoglycans (A-C), Verhoeff staining reagent for elastin (E-G), and Sirius red staining reagent for collagen (I-K). Scale bar: I00 $\mu$ m. Quantitative analysis of GAG (D), elastin $(\mathbf{H})$ and collagen $(\mathbf{L})$ content in vascular grafts $(n=3) . *$ indicated the graft lumen. $\star: P<0.05$, ns $=$ not significant. 
smooth, uniform and free of protrusions. Interestingly, the fiber diameter distribution of the fibrin vascular scaffolds (600-1600nm) was higher than that of the PU/fibrin vascular scaffolds (500-1200nm). This could be because the negative high voltage and the positively charged PU material surface electrostatically attracted each other during electrospinning, which caused the fibers of the PU/fibrin scaffold to stretch and become smaller in diameter.

Good mechanical properties are the necessary conditions for small-diameter blood vessel scaffolds. The mechanical properties of electrospun scaffolds were mainly determined by the properties of the scaffold, the interaction between the materials, and its geometric structure. $^{22,23}$ From the results, we found that the mechanical properties of the pure fibrin scaffolds were much lower than those of PU/fibrin vascular scaffolds. In addition, the tensile strength value of the PU/fibrin composite scaffolds increased from $3.45 \pm 0.32$ to $5.84 \pm 0.2 \mathrm{MPa}$, and the elongation at break value increased from $32.21 \pm 1.5 \%$ to $51.6 \pm 4.6 \%$. This may be because as the PU content increased, its macromolecular chain became longer and crossed with the fibrin molecular chain, and the interaction force between the two was enhanced, resulting in larger mechanical properties. However, the results of vascular scaffold modulus of elasticity indicated that the addition of PU could effectively change the elastic deformation ability of the composite scaffolds. Suture strength is an assessment of the ability of the suture to connect the graft to the surrounding tissue. According to related reports, the suture strength of small-diameter artificial blood vessels is about $0.5 \mathrm{~N} .^{24,25}$ In our study, it was found that when the PU content increased from $15 \%$ to $25 \%$, the suture strength values were $0.46 \mathrm{~N}$ and $0.58 \mathrm{~N}$, respectively, which indicated that the two groups of vascular scaffolds met the clinical requirements (Figure 2D). The greater the burst strength of the artificial blood vessel, the stronger the pressure resistance, and the better its reliability. From Figure 2E, we found that the burst pressure of PU/fibrin (15:85) and PU/fibrin (25:75) vascular scaffolds were very closed to the saphenous vein $(1680 \sim 2273 \mathrm{mmHg}){ }^{26}$ At the same time, they were much greater than the burst pressure of native blood vessels $(1500 \mathrm{mmHg}){ }^{27}$ This indicated that these scaffolds could be used as ideal candidates for artificial blood vessels. The hydrophilicity of the vascular scaffold is closely related to cell proliferation and adhesion. $^{28}$ The bigger water contact angle in PU/fibrin (25:75) vascular scaffold may be because PU is hydrophobic material, and its poor absorption performance is not conducive to contact with cells. Fibrin is a material with good hydrophilicity. Adding fibrin to the mixed material greatly improved the hydrophilicity of PU/fibrin, which was beneficial to cell growth.

Platelet adhesion often depends on the surface properties and hydrophilic strength of the material. ${ }^{29}$ The good results of the platelet adhesion test in the PU/fibrin vascular scaffolds may be due to the blend of fibrin with good hydrophilicity, which promoted the hydrophobic surface of the PU vascular scaffold to become a hydrophilic surface, thereby reducing the amount of platelet adhesion on the surface of the PU/fibrin scaffold. Various factors lead to damage to red blood cells, and the broken red blood cells can release hemoglobin. ${ }^{30}$ Thus, hemolysis rate is an indicator of the blood compatibility of the material. In our study, the hemolysis rate of the four PU/fibrin vascular scaffolds were less than the standard value of $5 \%$, which indicated that the vascular scaffolds would not undergo hemolysis. The coagulation index is also an important reference index. The results of the BCI values of the four $\mathrm{PU} /$ fibrin vascular scaffolds indicated that the change in PU content did not lead to change in the blood compatibility of the PU/fibrin vascular scaffold. We also confirmed from Figure $3 \mathrm{E}$ and $\mathrm{F}$ that the change in $\mathrm{PU}$ content did not affect the anticoagulant effect of the PU/ fibrin vascular scaffold.

The degradation performance of the scaffold material affects the regeneration and remodeling of vascular graft, and the best degradation rate should be consistent with the tissue regeneration rate. ${ }^{31}$ In this work, the mass loss of the four PU/fibrin scaffold materials in PBS increased with time, and the fibrin in the PU/fibrin scaffold accelerated its degradation rate (Figure 4A). Mostafa et $\mathrm{al}^{32}$ found that the biodegradation rate remained constant during the fibrin hydrogel degradation process at different times, and this constant biodegradation rate was beneficial to cell proliferation. At the same time, it was confirmed that the fibrin material had a high biodegradation rate. At 4 weeks, the residual mass of PU/fibrin (0:100) vascular scaffold was the highest (5.7\%). Moreover, the results in enzyme degradation experiments were similar to those in hydrolysis (Figure 4B). The degradation of the scaffold was observed by SEM, which further confirmed that the degradation rate of the scaffold material in the enzyme solution was significantly faster than that of PBS. With the increase of PU content, the degradability of the scaffold gradually decreased. Dong et $\mathrm{al}^{33}$ had reported that biological degradation performance was affected by many factors, such as 
polymer chain structure, crystallinity, ionic strength in the medium, enzyme activity and $\mathrm{pH}$ value. This may be because the molecular chain configuration of PU influenced its degradation performance. The enzyme activity in the lipase solution was significantly higher than in the PBS solution, thereby accelerating the degradation rate of the scaffold.

The tissue engineering vascular scaffold should promote cell adhesion and proliferation. Researchers have confirmed that electrospun scaffolds with nanomesh structure can better promote cell growth. ${ }^{34}$ Nafiseh et al ${ }^{35}$ evaluated the cell compatibility of electrospun PU/PCL tubular scaffolds, and the results showed that Vero cells could proliferate well on the electrospun scaffolds. To verify the feasibility of PU/fibrin vascular scaffolds, we used live cell fluorescence staining to observe cell adhesion and growth behavior. After 3 days and 5 days, we found that the number of MSCs living cells on the PU/fibrin (25:75) scaffold was significantly less than that on other groups of PU/fibrin scaffolds (Figure 5A). These results showed that the PU/fibrin (15:85) vascular scaffold had almost no toxic side effects and improved the adhesion and proliferation behavior of MSCs on the vascular scaffold. In addition, we used the CCK8 experiment for further verification. We found that the number of cells on four different PU/fibrin scaffolds increased with the co-culture time (Figure 5B). The results indicated that PU/fibrin vascular scaffold had good cell compatibility. The main component of scaffold material slowly degrades in vivo until finally replaced by collagen and other ECM. When MSCs are contracting or not growing, the vasoconstrictor function will be significantly improved. Therefore, we tested the ECM markers and SMC specificity at 6 days by quantitative RT-PCR. We found that the PU/fibrin (25:75) scaffold had the least expression of collagen, survivin and vimentin genes. This may be because the PU of the PU/fibrin vascular scaffold inhibited the secretion of the main components in the extracellular matrix.

It is necessary to pay attention to the thrombosis of vascular grafts for the successful construction of smalldiameter artificial vessels. There was no obvious thrombosis and vascular occlusion on the lumen of PU/fibrin vascular graft at three different time points (Figure 6A). Unexpectedly, it was found that there was artery-like neotissue on the surface of the PU/fibrin graft lumen at 3 months, which was very similar to the native artery (Figure 6B and C). The results confirmed that PU/fibrin vascular graft could be used as a material for artificial blood vessel scaffold. On the other hand, the risk index of thrombosis and occlusion during vascular scaffold implantation is the first key element of the evaluation. These results confirmed that the patency and viability in PU/fibrin grafts were better than those in PU grafts. Meanwhile, PU/fibrin grafts could effectively prevent the incidence of acute thrombosis and graft occlusion, and were significantly lower than in PU grafts (Figure 6D and E). This may be because PU/fibrin grafts with good degradation performance could change the tissue reaction and physicochemical properties, thereby reducing the risk of thrombosis and occlusion. Reconstruction of vascular grafts into functional neoartery is an ideal result of artificial blood vessels. Zhu et $\mathrm{al}^{36}$ designed a poly (L-lactideco- $\varepsilon$-caprolactone) vascular graft and implanted it in SD rats for 12 months. It was found that the graft formed a functional neoartery alike to the naive artery. In this article, the PU/fibrin group and the PU group showed significant functional differences in contraction and relaxation function, and the functionality of the PU/fibrin graft was very close to the native artery (Figure $6 \mathrm{~F}$ and $\mathrm{G}$ ). This may be because that PU with poor hydrophilicity and degradation rate affected the remodeling and regeneration of vascular grafts in vivo. Good mechanical properties can significantly improve vascular remodeling and regeneration. From our results, it was found that the elastic modulus, breaking strain and maximum stress in PU/fibrin at 3 months were similar to those of native arteries, but they were much lower than that in PU grafts (Figure 6H-J). In addition, the compliance index of the vascular graft can evaluate the successful transplantation of the artificial blood vessel into the host in vivo. The experimental results showed that the compliance of PU/fibrin vascular grafts at 1 month, 2 months and 3 months increased significantly (Figure 6K). These results further indicated that the PU/ fibrin graft conformed to an ideal artificial blood vessel scaffold.

Whether small-diameter artificial blood vessels can regenerate depends on whether vascular tunica media can be successfully reconstructed. Researchers had found that some factors such as the structural composition and mechanical strength of the vascular graft could affect the regeneration function of VSMC. ${ }^{37}$ In this work, we found that PU/fibrin samples showed a circular appearance arrangement at 3 months, and neointimal formation on the surface of the graft lumen (Figure 7A). This may be due to the electrospun PU/fibrin grafts with good fiber pore structure effectively improving the regeneration of vascular 
tunica media. Moreover, we also found that the expression of $\alpha$-SMA in PU/fibrin grafts was closed to that of native arteries, but it was much higher than that in PU grafts. The results indicated that $\mathrm{PU} /$ fibrin vascular grafts have successfully regenerated vascular functional neoarteries. On the one hand, the formation of a complete endothelial layer in vascular grafts can effectively reduce intimal hyperplasia and thrombosis. Researchers have used many methods to better optimize endothelialization of vascular grafts. ${ }^{38,39}$ Zhu et $\mathrm{al}^{40}$ used wet spinning and electrospinning techniques to prepare internal and external bi-layered vascular grafts. The results confirmed that the graft in vivo successfully completed regeneration into endothelial cells. Our results showed that the endothelial layer on the surface of PU/fibrin graft and PU graft was relatively thinner compared to the native artery (Figure 7A). However, the number of vWF cells in PU/fibrin grafts were more than in PU grafts. These results indicated that the PU/fibrin vascular graft regenerated the endothelial layer in vivo. On the other hand, some researchers have confirmed that macrophages as inflammatory mediators play important role in the process of vascular graft remodeling. ${ }^{41}$ The process of regeneration and remodeling of vascular grafts is related to inflammation. As an important inflammatory mediator, macrophages also participate in and regulate regeneration and remodeling. To design an ideal vascular graft, researchers had adopted a variety of methods, such as adding growth factors, changing the degradation performance of scaffold materials. ${ }^{42}$ In our work, PU/fibrin grafts with nanofibers arranging circumferentially not only effectively promoted cell adhesion and proliferation but also significantly hindered the polarization of cells. In addition, the number of CD68 positive cells in PU/fibrin graft was significantly less than that in PU graft. The results indicated that the degradation of PU/fibrin vascular grafts and compliance changed at 3 months after transplantation, thereby inducing the phenotype change of macrophages. The stable relationship between pro-inflammatory cytokines and anti-inflammatory cytokines is particularly important for tissue regeneration and reconstruction. We found that the secretion of inflammatory cytokines in PU/fibrin vascular grafts were lower than that in PU vascular grafts at 3 months. This may be due to the poor degradation and compatibility of pure PU, which affected the expression of related inflammatory factors. Importantly, the expression level of all inflammatory cytokines in the PU/fibrin graft at 3 months was closed to that in native arteries (Figure 7E-H). These results showed that the pro-inflammatory and anti-inflammatory cytokines have been maintained in an ideal state, which was conducted to vascular reconstruction and remodeling.

The composition of the ECM and the cells together determine the mechanical strength of the blood vessels. The formation of ECM deposits can compensate for the loss of mechanical strength during the degradation of vascular grafts, thereby bringing the new arteries closer to the mechanical strength of native arteries. ${ }^{43,44}$ Abundant ECM can effectively prevent vascular graft rupture during degradation and promote the successful regeneration of the graft in vivo. In our study, the distribution of ECM in the PU/fibrin grafts was circumferential arrangement. Concurrently, the density of ECM in PU/fibrin vascular grafts was obviously higher than that in PU grafts, but still slightly lower than in native arteries (Figure 8). After 3-month transplants, all vascular grafts did not rupture. We analyzed that PU/fibrin grafts that had not been completely degraded and ECM deposits worked together to ensure that the grafts resisted certain blood pressure.

\section{Conclusion}

In this article, we successfully prepared PU/fibrin vascular scaffold by electrospinning technology. The results confirmed that the fiber diameter of the PU/fibrin vascular scaffold was smaller than that of the PU scaffold. With the increase of PU content, the mechanical strength of PU/fibrin vascular scaffolds increased gradually. However, when the content of PU in PU/fibrin scaffold reached 25\%, the cell proliferation ability was significantly affected. Therefore, the PU/fibrin (15:85) vascular scaffold showed balanced mechanical strength, blood compatibility, degradability and cell compatibility. Moreover, we found that the function and mechanical strength of PU/fibrin (15:85) neoarteries was very closed to that of native arteries at 3 months after implantation. Concurrently, the cells amount of SMCs and ECs and ECM expression in PU/fibrin (15:85) vascular grafts at 3 months were significantly more than those of PU vascular grafts, but the macrophages were fewer. Overall, PU/ fibrin vascular grafts could be used as an ideal small-diameter tissue engineering vascular scaffold, and this research would promote progress of tissue engineering blood vessels field.

\section{Acknowledgments}

In this work, we have received strong financial support by Natural Science Foundation of Henan Province, Datong Applied Basic Research Program, the National Natural Science Foundation of China (81901874, 81903164), Zhengzhou Key Laboratory of Cardiac Structure 
Research (2019KFKT006), Postgraduate Research and Innovation Support Plan Project of Xinxiang Medical University (YJSCX201905Z), the College Student Innovation and Entrepreneurship Training Program Project of Henan Province (141039, 110411), Research and Innovation of College Students in Xinxiang Medical University (xyxskyz201948), Science and Technology Research Project (social development) of Henan Province in 2018 (182102310067), Independent Research Fund of Beijing in 2019, Plateau Discipline (Surgery) of the First Clinical College of Xinxiang Medical University, Opening Project Fund of Henan Key Laboratory of Neurological Repair (HNSJXF-2016-011), Novel Coronavirus Pneumonia Research and Development Project in 2020 of Medical Engineering College in Xinxiang Medical University (XYYXGC2020-005).

\section{Disclosure}

The authors declared that they have no conflicts of interest to this work. We declare that we do not have any commercial or associative interest that represents a conflict of interest in connection with the work submitted.

\section{References}

1. Wekesah FM, Kyobutungi C, Grobbee DE, et al. Understanding of and perceptions towards cardiovascular diseases and their risk factors: a qualitative study among residents of urban informal settings in Nairobi. BMJ Open. 2019;9(6):e026852. doi:10.1136/bmjopen-2018026852

2. Members WG, Mozaffarian D, Benjamin EJ, et al. Executive Summary: heart Disease and Stroke Statistics-2016 Update: A Report From the American Heart Association. Circulation. 2016;127(1):143-152.

3. Organization WH. World health statistics 2016: monitoring health for the SDGs, sustainable development goals. Geneva Switzerland Who. 2016;41:293-328.

4. Patterson JT, Gilliland T, Maxfield MW, et al. Tissue-engineered vascular grafts for use in the treatment of congenital heart disease: from the bench to the clinic and back again. Regen Med. 2012;7 (3):409-419.

5. Niklason LE. Readily Available Tissue-Engineered Vascular Grafts. Science. 2011;3(68):68ra9.

6. Chlupac J, Filova E, Bacakova L. Blood vessel replacement: 50 years of development and tissue engineering paradigms in vascular surgery. Physiological Res. 2009;58(2):S119-39.

7. Cleary MA, Geiger E, Grady C, et al. Vascular tissue engineering: the next generation. Trends Mol Med. 2012;18(7):394-404.

8. Shaikh FM, Callanan A, Kavanagh EG, et al. Fibrin: A Natural Biodegradable Scaffold in Vascular Tissue Engineering. Cells Tissues Organs. 2008;188(4):333-346.

9. Kang YH, Jeon SH, Park JY, et al. Platelet-rich fibrin is a Bioscaffold and reservoir of growth factors for tissue regeneration. Tissue Eng Part A. 2011;17(3-4):349-359.

10. Al Kayal T, Losi P, Pierozzi S, et al. A New Method for Fibrin-Based Electrospun/Sprayed Scaffold Fabrication. Sci Rep. 2020;10(1):5111. doi:10.1038/s41598-020-61933-z
11. Uttayarat P, Perets A, Li M, et al. Micropatterning of three-dimensional electrospun polyurethane vascular grafts. Acta Biomater. 2010;6(11):4229-4237. doi:10.1016/j.actbio.2010.06.008

12. Macocinschi D, Filip D, Vlad S. Natural-Based Polyurethane Biomaterials for Medical Applications. Biomaterials App Nanomed. 2011;309-332.

13. Pankajakshan D, Agrawal DK. Scaffolds in tissue engineering of blood vessels. Can J Physiol Pharmacol. 2010;88(9):855-873. doi:10.1139/Y10-073

14. Zhao L, Chen D, Yao Q, et al. Studies on the use of recombinant spider silk protein/polyvinyl alcohol electrospinning membrane as wound dressing. Int $J$ Nanomedicine. 2017;12:8103-8114. doi:10.2147/IJN.S47256

15. Liang Z, Xu Y, Meng H, et al. Preparation of spider silk protein bilayer small-diameter vascular scaffold and its biocompatibility and mechanism research. Composite Interfaces. 2014;21(9):869-884. doi:10.1080/15685543.2014.970416

16. Zhao L, Xu YL, Li M, et al. Preparation of bilayer spider silk protein vascular scaffold and its biomechanical properties and cell compatibility. J Med Biomechanics. 2013;28(5):559-566.

17. Hutmacher DW. Scaffold design and fabrication technologies for engineering tissues - state of the art and future perspectives. J Biomater Sci Polym Ed. 2001;12(1):107-124. doi:10.1163/ 156856201744489

18. Li S, Nih LR, Bachman H, et al. Hydrogels with precisely controlled integrin activation dictate vascular patterning and permeability. Nat Mater. 2017;16(9):953-961. doi:10.1038/nmat4954

19. Chandra P, Atala A. Engineering blood vessels and vascularized tissues: technology trends and potential clinical applications. Clin Sci (Lond). 2019;133(9):1115-1135. doi:10.1042/CS20180155

20. Ceccarelli J, Putnam AJ. Sculpting the blank slate: how fibrin's support of vascularization can inspire biomaterial design. Acta Biomater. 2014;10(4):1515-1523. doi:10.1016/j.actbio.2013.07.043

21. Zhang J, Wang Y, Liu C, et al. Polyurethane/polyurethane nanoparticle-modified expanded poly(tetrafluoroethylene) vascular patches promote endothelialization. $J$ Biomed Mater Res A. 2018;106(8):2131-2140. doi:10.1002/jbm.a.36419

22. Bin L, Lang H, Yin QL. Evaluation on tissue-engineered vascular scaffold materials. J Clin Rehabilitative Tissue Eng Res. 2010;14 (21):3883-3886.

23. Almeida HA, BáRtolo PJ. In Computer Simulation and Optimisation of Tissue Engineering Scaffolds: mechanical and Vascular Behaviour, ASME Biennial Conference on Engineering Systems Design \& Analysis, 2008, 8173-180

24. Asvar Z, Mirzaei E, Azarpira N, et al. Evaluation of electrospinning parameters on the tensile strength and suture retention strength of polycaprolactone nanofibrous scaffolds through surface response methodology. J Mech Behav Biomed Mater. 2017;75:369-378. doi:10.1016/j.jmbbm.2017.08.004

25. Chaparro FJ, Matusicky ME, Allen MJ, et al. Biomimetic microstructural reorganization during suture retention strength evaluation of electrospun vascular scaffolds. J Biomed Mater Res B Appl Biomater. 2016;104(8):1525-1534.

26. Konig G, Mcallister TN, Dusserre N, et al. Mechanical properties of completely autologous human tissue engineered blood vessels compared to human saphenous vein and mammary artery. Biomaterials. 2009;30(8):1542-1550. doi:10.1016/j.biomaterials.2008.11.011

27. Gao J, Chen S, Tang D, Jiang L, Shi J, Wang S. Mechanical Properties and Degradability of Electrospun PCL/PLGA Blended Scaffolds as Vascular Grafts. Transactions Tianjin Univ. 2018;25 (2):152-160. doi:10.1007/s12209-018-0152-8

28. Nguyen TH, Padalhin AR, Seo HS, et al. A hybrid electrospun PU/ PCL scaffold satisfied the requirements of blood vessel prosthesis in terms of mechanical properties, pore size, and biocompatibility. J Biomater Sci Polym Ed. 2013;24(14):1692-1706. doi:10.1080/ 09205063.2013.792642 
29. Barbucci R, Lamponi S, Aloisi AM. Platelet adhesion to commercial and modified polymer materials in animals under psychological stress and in a no-stress condition. Biomaterials. 2002;23(9):1967-1973. doi:10.1016/S0142-9612(01)00323-4

30. Alexandre N, Ribeiro J, Gartner A, et al. Biocompatibility and hemocompatibility of polyvinyl alcohol hydrogel used for vascular grafting-In vitro and in vivo studies. J Biomed Mater Res A. 2014;102(12):4262-4275.

31. Zhang L, Liu X, Li G, et al. Tailoring degradation rates of silk fibroin scaffolds for tissue engineering. J Biomed Mater Res A. 2019;107 (1):104-113.

32. Soleimannejad M, Ebrahimi-Barough S, Soleimani M, et al. Fibrin gel as a scaffold for photoreceptor cells differentiation from conjunctiva mesenchymal stem cells in retina tissue engineering. Artif Cells, Nanomed Biotechnol. 2018;46(4):805-814. doi:10.1080/21691401. 2017.1345922

33. Dong Y, Liao S, Ngiam M, et al. Degradation Behaviors of Electrospun Resorbable Polyester Nanofibers. Tissue Eng Part B Rev. 2009;15(3):333-351. doi:10.1089/ten.teb.2008.0619

34. Boccafoschi F, Habermehl J, Vesentini S, et al. Biological performances of collagen-based scaffolds for vascular tissue engineering. Biomaterials. 2005;26(35):7410-7417. doi:10.1016/j.biomaterials. 2005.05.052

35. Jirofti N, Mohebbi-Kalhori D, Samimi A, et al. Small-diameter vascular graft using co-electrospun composite PCL/PU nanofibers. Biomed Mater. 2018;13(5):055014. doi:10.1088/1748-605X/aad4b5

36. Zhu M, Yifan W, Wen L, et al. Biodegradable and elastomeric vascular grafts enable vascular remodeling. Biomaterials. 2018;183: 306-318.
37. Qiu J, Zheng Y, Hu J, et al. Biomechanical regulation of vascular smooth muscle cell functions: from in vitro to in vivo understanding. $J$ R Soc Interface. 2013;11(90):20130852. doi:10.1098/rsif.2013.0852

38. Zeng W, Yuan W, Li L, et al. The promotion of endothelial progenitor cells recruitment by nerve growth factors in tissue-engineered blood vessels. Biomaterials. 2010;31(7):1636-1645. doi:10.1016/j. biomaterials.2009.11.037

39. Melchiorri AJ, Hibino N, Fisher JP. Strategies and Techniques to Enhance the In Situ Endothelialization of Small-Diameter Biodegradable Polymeric Vascular Grafts. Tissue Engineering Part B: Reviews. 2013;19(4):292-307. doi:10.1089/ten.teb.2012.0577

40. Zhu M, Wang Z, Zhang J, et al. Circumferentially aligned fibers guided functional neoartery regeneration in vivo. Biomaterials. 2015;61:85-94.

41. Roh JD, Sawh-Martinez R, Brennan MP, et al. Tissue-engineered vascular grafts transform into mature blood vessels via an inflammation-mediated process of vascular remodeling. Proc Natl Acad Sci U S A. 2010;107(10):4669-4674.

42. Pashneh-Tala S, Macneil S, The Tissue-Engineered CF. Vascular Graft_-Past, Present, and Future. Tissue Eng Part B Rev. 2016;22 (1):68-100.

43. Syedain Z, Reimer J, Lahti M, et al. Tissue engineering of acellular vascular grafts capable of somatic growth in young lambs. Nat Commun. 2016;7:12951.

44. Li W, Chen J, Xu P, et al. Long-term evaluation of vascular grafts with circumferentially aligned microfibers in a rat abdominal aorta replacement model. J Biomed Mater Res B Appl Biomater. 2018;106 (7):2596-2604.
International Journal of Nanomedicine

\section{Publish your work in this journal}

The International Journal of Nanomedicine is an international, peerreviewed journal focusing on the application of nanotechnology in diagnostics, therapeutics, and drug delivery systems throughout the biomedical field. This journal is indexed on PubMed Central, MedLine, CAS, SciSearch ${ }^{\circledR}$, Current Contents ${ }^{\circledR} /$ Clinical Medicine,

\section{Dovepress}

Journal Citation Reports/Science Edition, EMBase, Scopus and the Elsevier Bibliographic databases. The manuscript management system is completely online and includes a very quick and fair peer-review system, which is all easy to use. Visit http://www.dovepress.com/ testimonials.php to read real quotes from published authors. 